\title{
A redistribution method for axisymmetric diffusion
}

\author{
S. Shankar and A. F. Ghoniem
}

\author{
Department of Mechanical Engineering \\ Massachusetts Institute of Technology \\ Cambridge, Massachusetts, 02139, U.S.A. \\ subram@centaurus.mit.edu \\ ghoniem@mit.edu
}

\begin{abstract}
We develop grid-free numerical procedures to compute axisymmetric incompressible flows. In particular, we formulate a grid-free 'redistribution method' to handle diffusion processes accurately on a disordered collection of computational elements. We validate the numerical procedures by computing the Stokes flow and the Navier-Stokes flow of a vortex ring.
\end{abstract}

\section{Introduction}

Vortex methods simulate fluid flows by following a collection of computational elements ('vortex elements') which transport of vorticity, and other conserved scalars such as energy and chemical species $[4,6,7]$. Often, those quantities are transported due to convection and diffusion. The convection process is handled accurately in a grid-free manner by moving the computational points according to the local velocity field. The diffusion process must also be implemented accurately using grid-free procedures to maintain the advantages of a Lagrangian computation.

In this paper we formulate grid-free methods to compute the evolution of the vorticity and temperature for axisymmetric incompressible flows in free-space. A fractional-step method $[2,5]$ is used to solve the governing equations. In this context, the diffusive effects are governed by the unsteady Stokes equations. To compute the Stokes equations accurately on a disordered distribution of computational elements is our primary objective.

We present the governing equations in Section 2. In Section 3 we formulate a vortex method to solve those equations. In Section 4 we briefly describe the extension of the redistribution method [11] to solve the Stokes equations for the vorticity and temperature in an axisymmetric domain; details of the formulation are given in [9]. In Section 5 we discuss the properties of the method. The numerical procedures are validated in Section 6 by computing the Stokes flow and the Navier-Stokes flow of a vortex ring. Conclusions and further work are discussed in Section 7.

\section{Governing equations}

We consider the free-space flow of a homogenous, incompressible fluid of constant density in an axisymmetric domain; we assume that the kinematic viscosity, $\nu$, and the thermal diffusivity, $\kappa$, are constant. Let $r$ and $z$ be the radial and axial directions of the flow in the $r-z$ plane so that $-\infty<r, z<\infty$. The location of a point in this plane is denoted by $\vec{x}=(r, z)=r \hat{r}+z \hat{z}$; the symbols $\hat{r}$ and $\hat{z}$ are the unit vectors. Let $t$ be the time. The velocity field is denoted by $\vec{u}(\vec{r}, t)=\left(u_{r}, u_{z}\right)=u_{r}(\vec{r}, t) \hat{r}+u_{z}(\vec{r}, t) \hat{z}$. The vorticity field, which is the curl of the velocity, is denoted by $\omega(\vec{r}, t)$; it points in the direction $\hat{z} \times \hat{r}$ into the plane. Let $T(\vec{r}, t)$ be the temperature that is transported as a passive scalar; since the density and specific heat are 
constant, the temperature is proportional to the internal energy of the fluid. The governing equations for the vorticity and temperature are $[1,3]$ :

$$
\begin{gathered}
\frac{1}{r} \frac{\partial\left(r u_{r}\right)}{\partial r}+\frac{\partial u_{z}}{\partial z}=0 \\
\frac{D \omega}{D t}=\frac{\omega u_{r}}{r}+\nu\left(\frac{\partial^{2} \omega}{\partial r^{2}}+\frac{1}{r} \frac{\partial \omega}{\partial r}-\frac{\omega}{r^{2}}+\frac{\partial^{2} \omega}{\partial z^{2}}\right) \\
\omega \equiv \frac{\partial u_{r}}{\partial z}-\frac{\partial u_{z}}{\partial r}, \\
\frac{D T}{D t}=\kappa\left(\frac{\partial^{2} T}{\partial r^{2}}+\frac{1}{r} \frac{\partial T}{\partial r}+\frac{\partial^{2} T}{\partial z^{2}}\right)
\end{gathered}
$$

where

$$
\frac{D}{D t} \equiv \frac{\partial}{\partial t}+\left(u_{r} \frac{\partial}{\partial r}+u_{z} \frac{\partial}{\partial z}\right)
$$

is the material derivative.

To solve the above equations, the initial vorticity and the temperature distributions must be specified. The boundary conditions along the axis of symmetry are discussed in Section 4 . In the next section we formulate a vortex method to solve the above equations numerically.

\section{Vortex method}

The vortex method presented here is based on a fractional-step algorithm [2, 5], that separates the inviscid and viscous processes at each time-step. To do this, the conservation equations (2) and (4) are approximated by the following two steps:

\section{Inviscid step:}

$$
\begin{aligned}
\frac{d \vec{X}}{d t} & =\vec{u} \\
\frac{d \Gamma_{\omega}}{d t} & =0 \\
\frac{d \Gamma_{T}}{d t} & =0
\end{aligned}
$$

Viscous step:

$$
\begin{aligned}
& \frac{d \vec{X}}{d t}=0 \\
& \frac{\partial \omega}{\partial t}=\nu\left(\frac{\partial^{2} \omega}{\partial r^{2}}+\frac{1}{r} \frac{\partial \omega}{\partial r}-\frac{\omega}{r^{2}}+\frac{\partial^{2} \omega}{\partial z^{2}}\right) \\
& \frac{\partial T}{\partial t}=\kappa\left(\frac{\partial^{2} T}{\partial r^{2}}+\frac{1}{r} \frac{\partial T}{\partial r}+\frac{\partial^{2} T}{\partial z^{2}}\right)
\end{aligned}
$$

In the above equations, $\vec{X}$ is the location of a fluid element, $\vec{u}$ is its velocity,

$$
\Gamma_{\omega} \equiv \iint \omega d r d z
$$

is the circulation, and

$$
\Gamma_{T} \equiv \iint \operatorname{Tr} d r d z
$$


is the internal energy per unit mass divided by the specific heat. The integrals in (12) and (13) are taken over the area of the fluid element in the half-plane $0 \leq r$. For the Stokes equations, the circulation of an element decays in time, while the energy is still conserved.

Equations (7) and (8) are obtained by integrating over the half-plane the equations (2) and (4) without the diffusion terms. The velocity is obtained from the vorticity ([1], eqn. 2.4.10).

To solve (6) through (11) using a vortex method, the initial vorticity and temperature are first represented by computational elements. To do this, we divide the flow region into small cells, and assign an element to each cell. The mathematical representation is then

$$
\begin{aligned}
\omega(\vec{x}, 0) & =\sum_{i=1}^{N} \Gamma_{\omega_{i}}(0) \delta\left(\vec{x}-\vec{x}_{i}(0)\right), \\
T(\vec{x}, 0) & =\sum_{i=1}^{M} \Gamma_{T_{i}}(0) \delta\left(\vec{x}-\vec{x}_{i}(0)\right),
\end{aligned}
$$

where, $i$ is the index for the element, $\vec{x}_{i}(t)$ is its position at time $t, \Gamma_{\omega_{i}}(t)$ is its circulation, $\Gamma_{T_{i}}(t)$ is its internal energy, and $\delta(\cdot)$ is the Dirac delta function. According to equations (12) and (13), the initial circulation of an element is taken to be the vorticity at the element location times the area of the cell; the initial internal energy is the product of the temperature at the element location, the radial location of the element and the cell area. Each computational element, however, need not carry both the circulation and energy, although saving in computational time may be achieved if they did.

During the inviscid step, we move each computational element according to the local velocity field (6) while keeping its circulation and energy unchanged $(7,8)$. The velocity of each element is computed by summing up all the velocities induced at its location by all the vorticity carrying elements including itself ([1], eqns. 2.2.11 \& 7.2.13). The velocity is desingularized [5, 13] using a small smoothing parameter of size $\sqrt{0.5 \nu \Delta t}$, where $\Delta t$ is the time-step in the computation.

At the end of the inviscid step, we perform the viscous step to account for diffusion. During the viscous step we do not move the computational elements (9), but change their circulations (10) and the energies (11) using the redistribution method. We formulate the redistribution method in the next section to solve the Stokes equations (10) and (11).

We reverse the sequence of the inviscid and viscous steps at every time-step to improve accuracy [2].

\section{Redistribution method}

The effect of diffusion is simply to spread out the circulation and energy of each computational element. The redistribution method is based on this idea: it spreads the circulation and energy of an element to its neighboring elements located within a chosen radius, $R$, according to the Stokes equations. This radius is proportional to the diffusion length scale $h_{\lambda}=\sqrt{\lambda \Delta t}$; the diffusivity $\lambda$ being either the kinematic viscosity or thermal diffusivity, and $\Delta t$ is the time-step. In our computations, the average distance between the computational elements is taken to be $\sqrt{8} h_{\lambda}$; and the neighborhood radius $R$ is taken to be $4 h_{\lambda}$. These two parameters are chosen so that a solution to the system of equations, derived below, becomes possible [11].

More precisely, let $i$ be an element located at $\left(r_{i}, z_{i}\right)$, whose circulation (or energy) needs to be diffused, and $j=1, \ldots, m$ be its neighboring elements located within the radius $R$. Let $f_{i j}^{n}$ be the fraction of the circulation (or energy) moved from $i$ to its neighbor $j$ to advance the vorticity (or temperature) from time level $n$ to the next time level $n+1$. Then the vorticity field at time level $n$,

$$
\omega^{n}=\sum_{i} \Gamma_{i}^{n} \delta\left(\vec{x}-\vec{x}_{i}\right)
$$

becomes 


$$
\omega^{n+1}=\sum_{i} \sum_{j} f_{i j}^{n} \Gamma_{i}^{n} \delta\left(\vec{x}-\vec{x}_{j}\right)
$$

at the next time level.

Now the question is how do we find those fractions $f_{i j}^{n}$. To do that, we will ensure that the Fourier modes of the numerical solution are correctly damped [11]. This is accomplished by matching the Hankel-Fourier transform [12] of the the numerical solution (17) given by

$$
\hat{\omega}^{n+1}=\sum_{i} \sum_{j} f_{i j}^{n} \Gamma_{i}^{n} r_{j} J_{1}\left(k_{r} r_{j}\right) e^{-i k_{z} z_{j}}
$$

with that of the exact solution

$$
\hat{\omega}_{e}^{n+1}=\sum_{i} \Gamma_{i}^{n} r_{i} J_{1}\left(k_{r} r_{i}\right) e^{-i k_{z} z_{i}} e^{-k^{2} \nu \Delta t}
$$

to desired order of accuracy $O\left(h_{\lambda}^{M}\right)$, as described below; and $M$ is a positive integer. The exact solution (19) is obtained by solving the Stokes equation (10) with initial vorticity (16). In the above equations, $J_{1}(\cdot)$ is the Bessel function of first kind and order one; and the wave number is denoted by $\vec{k}=k_{r} \hat{r}+k_{z} \hat{z}$, and its magnitude by $k$. For the diffusion of internal energy, the above procedure is the same except that the radial modes are now Bessel functions of first kind and order zero.

We next expand (18) and (19) using Taylor's series around $\vec{x}_{i}$ for small times and ensure that the truncation errors vanish to desired accuracy; the distances between the vortices within the neighborhood are scaled by $h_{\lambda}$. This results in a system of linear equations for the fractions $f_{i j}^{n}$ :

$$
\begin{aligned}
& \sum_{j} f_{i j}^{n}=1 ; \\
& \sum_{j} f_{i j}^{n} \Delta r_{i j}=\frac{\varepsilon}{r_{i} / h_{\lambda}}, \\
& \sum_{j} f_{i j}^{n} \Delta z_{i j}=0 ; \\
& \sum_{j} f_{i j}^{n} \Delta r_{i j} \Delta r_{i j}=2, \\
& \sum_{j} f_{i j}^{n} \Delta r_{i j} \Delta z_{i j}=0 \\
& \sum_{j} f_{i j}^{n} \Delta z_{i j} \Delta z_{i j}=2,
\end{aligned}
$$

where $\Delta \xi_{i j} \equiv\left(\xi_{j}-\xi_{i}\right) / h_{\lambda}$. The equations (20)-(25) are $O\left(h_{\lambda}\right)$ approximation to the Stokes equations (10) and (11). Higher-order spatial accuracy can be achieved by including additional redistribution equations that are obtained by retaining higher-order terms in the Taylor's series expansion.

The value of the parameter $\varepsilon$ in $(21)$ is -1 for the diffusion of circulation, and 1 for the diffusion of energy. When $\varepsilon=0$, however, the same equations represent the diffusion of circulation and energy in Cartesian two-dimensional flows. The above redistribution equations maintain the conservation laws of the Stokes equations exactly.

The above system of linear equations is usually underdetermined, since the number of elements within the neighborhood is often more than the number of equations. There is, however, a restriction on the values of the fractions $f_{i j}^{n}$. For stability, all fractions must be nonnegetive. The linear system can be solved using any standard simplex method program from IMSL for example. When no nonnegetive solution can be found, it is an indication that 'holes' may be present in the distribution of the computational elements. These holes are filled 
until an acceptable solution is obtained. This automatic addition of computational elements maintains the chosen resolution at all times, and further ensures that the vorticity diffuses correctly into the surrounding irrotational flow.

In our computations, we use elements only in the half-plane $0 \leq r$. Then the axis of symmetry is a boundary of the computational domain, and appropriate boundary conditions must be applied there. To do so, we place a layer of 'mirror elements' on the negetive side of $r$. The mirror elements are simply reflections of the elements on the positive side, whose radial locations are less than the neighborhood radius $R$ mentioned above.

The vorticity is antisymmetric in $r$, and hence vanishes on the axis. Therefore while diffusing the vorticity of the elements near the axis, the amounts of circulation moved to the mirrors are considered 'lost'. This ensures the correct decay of the circulation the half-plane.

The temperature is symmetric in $r$, and hence there is no heat flux across axis. Therefore, the energy received by a mirror element is given back to its mirror located on the positive side. This conserves the energy in the half-plane.

We must also point out a numerical difficulty caused by the equation (21): the righthand side becomes singular whenever the element to be diffused is very close to the axis. To avoid this singularity, we keep a thin strip near the axis free of computational elements. Of course, convection could still move some of the elements inside this strip due to numerical errors. However, those elements are moved back onto the edge of the strip and at the same time maintaining the appropriate conservation laws. The numerical results presented in the next section show that these procedures indeed reproduce the vorticity and temperature fields accurately.

We next discuss the theoretical properties of the method.

\section{Properties of the redistribution method}

In this section, we give a summary the properties of the method. Further discussion of the following properties is given in $[9,11]$. The advantages of the method achieved for cartesian two-dimensional flows successfully carry over to the axisymmetric case as well.

1. The redistribution equations spread the conserved quantities over only a finite number of neighbors inside a small area. This allows the method to resolve sharp gradients in the flow fields accurately [10].

2. The redistribution method does not require a uniform distribution of computational elements, or even an ordering of the elements. In particular, even if convection disorders the initial uniformity of the elements, the adaptive addition of new elements allows the computation to continue without loss of accuracy.

3. The redistribution method does not use any smoothing function to perform diffusion; the resulting desireable properties, especially the resolution of short scales, are discussed in [11], Sec. 9.1.

4. The conservation laws of the Stokes equations are exactly maintained using only a finite number of elements within the neighborhood, even if the elements are disordered.

5. The sign of a uniformly positive or negative vorticity field is preserved for any finite order of accuracy by the method, at least for the Stokes equations ([11], Sec. 9.5).

6. The redistribution method can, in principle, have any order of accuracy. At the same time, the splitting error of the fractional-step algorithm must be considered for spatial accuracy beyond fourth-order.

The advantages of the above properties have been demonstrated for the computation of two-dimensional unsteady separated flows at high Reynolds numbers [10]. In the next section we present numerical results to verify that those properties can be achieved for axisymmetric flows also. 


\section{Numerical results}

To validate the method, we computed the Stokes flow and the Navier-Stokes flow of a vortex ring. We present the numerical results in the next two subsections. We verified the results using various time-steps and corresponding spatial resolutions. The results presented here correspond to the smallest time-step used in our convergence study. The computations were carried out in single-precision on SGI/IRIX-6.1 with an average speed of $15 \mathrm{Mflops}$ and with a machine zero of about $6.0 \times 10^{-8}$.

\subsection{Stokes flow}

The redistribution method is tested on the Stokes flow due to a point source of circulation and energy. For these two cases, exact solutions are available that are simply the free-space Green's functions of the Stokes equations (10) and (11).

For the diffusion of vorticity, an initial point source of unit circulation, represented by a single computational element, is placed at $(r, z)=(2.5,0.0)$. The time-step is $\Delta \tau=0.004$, with the corresponding spatial resolution $R \approx 0.2530$. The computation was continued until time $\tau=1.0$ when the circulation in the half-plane had decayed to about eighty percent of the initial value; by this time it was clear that the handling of the axis boundary condition is accurate. The number of computational elements is about 3600 at this time. The impulse is conserved with a relative error less than $10^{-6}$ at all times, and the axial center of vorticity is conserved with a relative error less than $10^{-5}$. At the end of the computation, the relative error in mean square axial expansion is about $2.55 \times 10^{-3}$, and the error in the circulation in the half-plane is about $1.17 \times 10^{-3}$. More importantly, the high wave numbers that are most susceptible to dissipative errors are also correctly damped: the relative error in the maximum vorticity at $\tau=0.50$ is about $2.47 \times 10^{-4}$, and at $\tau=1.00$ the error is about $3.47 \times 10^{-4}$. In figure $1(\mathrm{a})$ the computed vorticity contours at time $\tau=0.50$ are compared with the exact solution.

For the diffusion of heat, an initial point source of unit internal energy, represented by a single computational element, is placed at $(r, z)=(2.5,0.0)$. The time-step and spatial resolution is the same as that for the vorticity. The computation is performed until time $\tau=1.30$, long enough to verify that the axis boundary condition works correctly; at this time, the maximum temperature is already attained on the axis. The number of computational elements is about 4100 at this time. The energy in the half-plane is conserved to machine precision. The axial center of temperature is conserved with a relative error less than $10^{-6}$ at all times. The mean square radial and axial expansions of the temperature field grow linearly with time as predicted theoretically, and the relative error in both is less than $10^{-5}$ throughout the computation. Further, the relative error in the maximum vorticity at $\tau=0.70$ is about $1.66 \times 10^{-3}$ and at $\tau=1.30$ the error is about $4.43 \times 10^{-4}$. The higher error at the earlier time is due to the high gradients arising from the transition of the temperature from a local minimum on the axis to the maximum value at the source location. In figure 1(b) the computed temperature contours at time $\tau=0.70$ are compared with the exact solution.

The numerical errors in the above conserved quantities are primarily due to round-off errors, and due to the truncation of the exponentially small vorticity and temperature fields determined a priori by a cut-off value of circulation and energy. The circulation or the energy of an element is not diffused if it falls below this cut-off; in the computations, a cut-off value of $10^{-6}$ is used.

In the next subsection we include convection as well to verify that the numerical procedures can handle the flow just as accurately as it did for the Stokes flow.

\subsection{Navier-Stokes flow}

When convection is present, the computational elements become disordered due to the straining of the flow. In this section we show that the method still maintains high accuracy under these conditions. As a nontrivial test case, we compute the Navier-Stokes flow of the propagation of a vortex ring in free-space. At the same time, we wanted to verify that our vortex method produces the pointwise values of the vorticity and temperature fields correctly at all times. Since 
we did not find suitable data in the literature, we computed this flow using a finite-difference method also. A convergence study of the finite-difference solution was performed based on the mesh-size, time-step, and domain size. In our finite-difference computations, we verified the convergence, not only of the various global moments of vorticity and temperature fields, but also the poinwise values of the fields themselves at different times. The best resolution used as many as $512 \times 1024$ computational points distributed uniformly over the domain $0 \leq r \leq 8$, $-8 \leq z \leq 8 ;$ and a time-step of 0.0005 .

The redistribution computation starts from a vortex ring of small core located radially a unit distance from the axis of symmetry. The ring has unit circulation and unit energy. The Reynolds number is $R e=50$ based on the initial circulation and ring radius. The Prandtl number is $\operatorname{Pr}=1$.

The initial vorticity distribution over the core of the vortex ring is obtained by allowing a point source to diffuse out as a Stokes flow (no convection) until the scaled time $\tau \equiv t / R e=$ 0.002. At this time, the vorticity distribution is almost a two-dimensional Gaussian with a core radius of about 0.18 ; just outside the core, the vorticity is less than one percent of the maximum. The initial temperature distribution is obtained similarly.

The figure 2 shows the distribution of computational elements. Notice that the points are disordered and denser near the center of the vortex due to the straining of the flow. New elements are automatically added only where the conserved quantities spread out. We replaced elements very close to each other, located within a radius $\sqrt{0.5 \Delta \tau}$, by a single element [10]. The scaled time-step $\Delta \tau$ is 0.0005 , and the corresponding spatial resolution $R \approx 0.0894$. The small circle in figure 1(c) indicates the size of the spatial resolution. The number of computational elements initially is about 400 , and grow almost linearly to roughly 5000 elements at scaled time $\tau=0.15$. At this time, the circulation in the half-plane had reduced to about eighty percent of its initial value.

The figure 3 provides a clear evidence of the accuracy of the method: it correctly reproduces the vorticity and temperature fields. We also verified that they are correct at several intermediate times. The vorticity is obtained by convolving the circulations with an infinite-order axisymmetric smoothing function; the temperature is obtained similarly. Notice in figure 3(a) that the vorticity and temperature fields have evolved very differently although they were identical initially; the maximum temperature is attained on the axis, while the vorticity vanishes there. The impulse of the vortex ring is conserved with a relative error less than $2.0 \times 10^{-5}$ at all times. The internal energy in the half-plane is conserved with a relative error less than $4.0 \times 10^{-5}$. The relative error in the circulation is less than $2.11 \times 10^{-3}$. The relative error in the velocity of the vortex ring [8] is about $2.35 \times 10^{-4}$ at the end of the computation.

The accuracy of the above numerical results is excellent considering that the computational elements are disordered and that a relatively coarse spatial resolution was used in the computations.

\section{Conclusions}

We have developed numerical procedures that are truly mesh-free for computing axisymmetric flows. The axisymmetric redistribution method simulates diffusion accurately even if convection causes strong distortions in the distribution of computational elements. The ability of the method to satisfy various conservation laws exactly by construction, together with other properties, provide reliable and accurate simulations of more complex flow fields. Infact, we are developing these procedures for the direct numerical simulation of jet diffusion flames. The redistribution method is an explicit time-stepping scheme, and we are working to obtain an implicit formulation to relax stability restrictions. The procedures can also be used to simulate many reacting flows based on coupling functions [14]. To compute an even wider class of flows, however, we need to handle diffusivities that vary spatially; that is also necessary to compute incompressible flows based on large eddy simulations. In this direction, our preliminary numerical results for one-dimensional diffusion are just as accurate as the constant diffusivity computations. 


\section{Acknowledgements}

This work was supported by the US Department of Energy, Basic Energy Sciences, mics, DE-F602-98ER25355. S. Shankar appreciates the helpful discussions with Professor L. van Dommelen, Department of Mechanical Engineering, FAMU-FSU College of Engineering.

\section{References}

1 Batchelor, G. K.: An Introduction to Fluid Dynamics (Cambridge Univ. Press, Cambridge), (1987).

2 Beale, J. T. \& Majda, A.: Rates of convergence for viscous splitting of the Navier-Stokes equations, Math. Comput. 37, (1981), 243-259.

3 Bird, R. B., Stewart, W. E., \& Lightfoot, E. N.: Transport phenomena (John Wiley, New York), (1960).

4 Chorin, A. J.: Vortex Methods, PAM Report 593, Department of Mathematics, University of California, Berkeley, (1993).

5 Chorin, A. J.: Numerical study of slightly viscous flow, J. Fluid Mech. 57, (1973), 785-796.

6 Ghoniem, A. F.: Computational methods in turbulent reacting flow, in Lectures in Applied Mathematics, Vol. 24, edited by G. S. S. Ludford, (American Mathematical Society, Providence), (1986), 199-265.

7 Leonard, A.: Vortex methods for flow simulation, J. Comput. Phys. 37, (1980), 289-335.

8 Saffman, P. G.: The velocity of viscous vortex rings, Stud. Appl. Math. 49, (1970), 371-380.

9 Shankar, S. \& Ghoniem, A. F.: A grid-free vortex method for axisymmetric flows, to be submitted to J. Comput. Phys.

10 Shankar, S.: A new mesh-free vortex method, Ph.D. thesis, Florida State University, 1996.(unpublished).

11 ShankaR, S. \& van Dommelen, L.: A new diffusion procedure for vortex methods, J. Comput. Phys. 127, (1996), 88-109.

12 Sneddon, I. N.: The use of integral transforms (McGraw-Hill, New York), (1972).

13 van Dommelen, L. \& Rundensteiner, E. A.: Fast, adaptive summation of point forces in the two-dimensional Poisson equation, J. Comput. Phys. 83, (1989), 126-147.

14 Williams, F. A.: Combustion theory (Addison-Wesley, New York), (1985). 

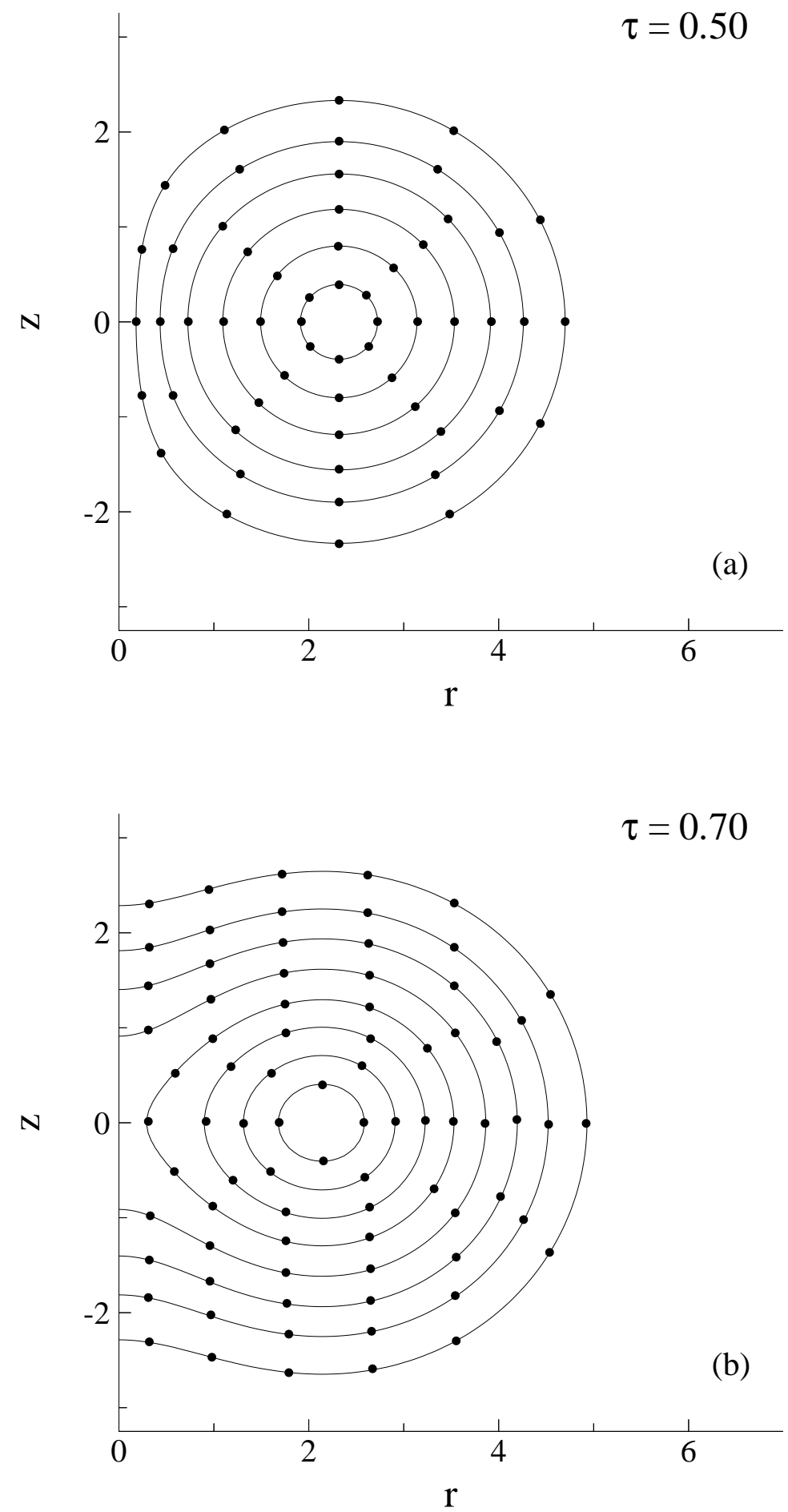

Figure 1: Stokes flow of point source: (a) Vorticity contours at $\tau=0.50: \omega=0.010,0.025,0.045$, $0.075,0.110,0.140$. (b) Temperature contours at $\tau=0.70: T=0.010,0.020,0.032,0.048,0.067$, $0.085,0.102,0.115$. Solid lines are exact and symbols are redistribution solutions. 

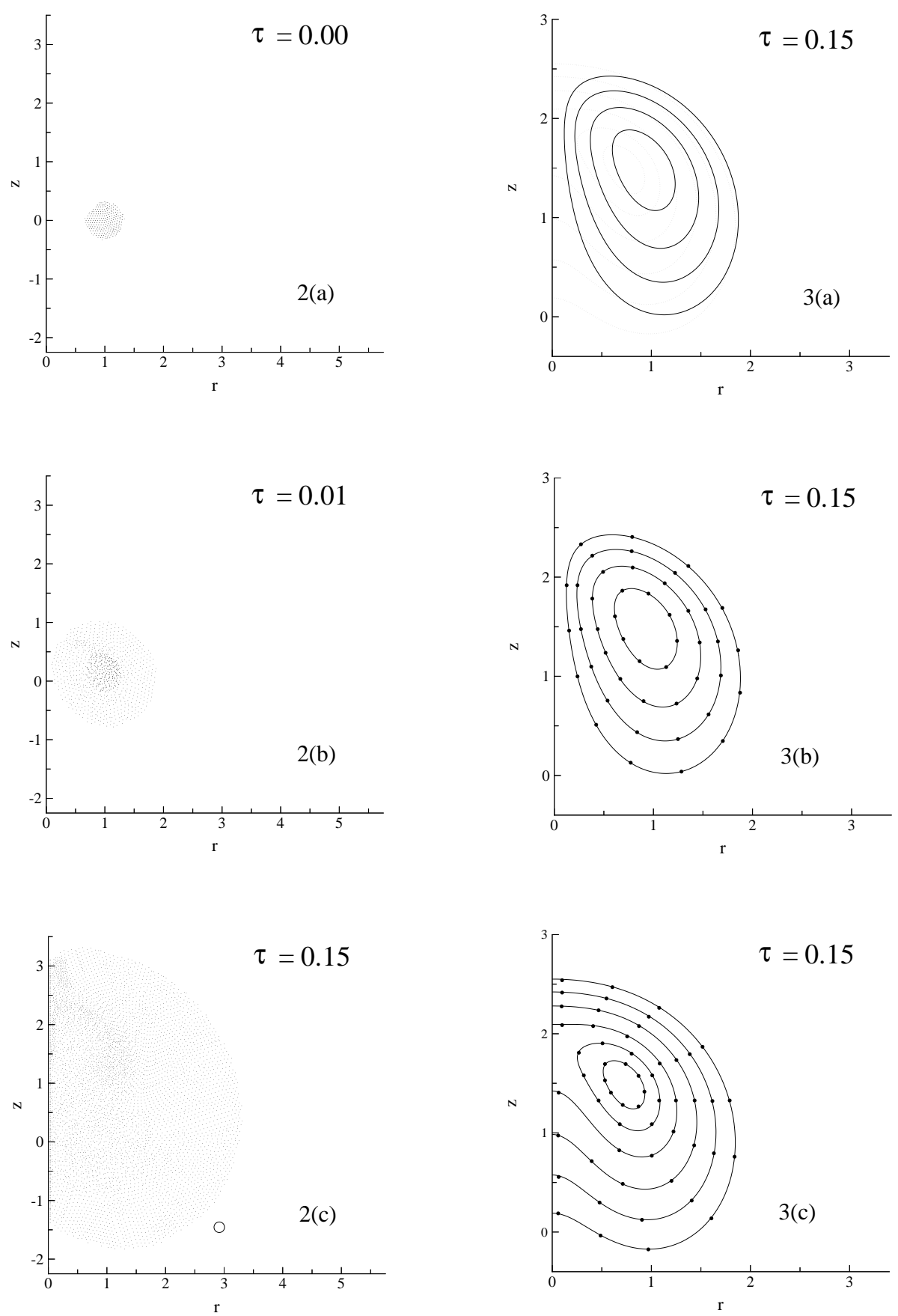

Figure 2: Computational elements for the flow of a vortex ring at $\operatorname{Re}=50$ and $\operatorname{Pr}=1$. The small circle in 2(c) represents the spatial resolution.

Figure 3: (a) Solid lines are vorticity contours, $\omega=0.08,0.15,0.25,0.375$; dotted lines are temperature contours, $T=0.08,0.15,0.25,0.375,0.475,0.54$. (b) \& (c) are vorticity and temperature contours; solid lines are finite-difference solutions and symbols are redistribution solutions. 